\title{
Vasculitispauciimmun Following Silicone Exposure from Breast Implantation
}

\author{
Awatef Azzebi, Dorsaf Zallema, Wissal Sahtout, Narjes Ben Aicha, Yosra Guedri, Sanda Mrabet, Asma Fradi, \\ Samira Ben Amor, Ferdaoues Sabri, Moncef Mokni and Abdellatif Achour
}

Department of Nephrology Dialysis and Renal Transplantation, Sahloul Hospital, Sousse, France

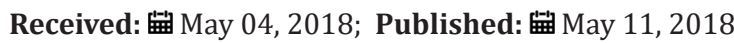

*Corresponding author: Narjes Ben Aicha, Department of Dialysis and Renal Transplantation, Sahloul Sospital, Sousse, France

\begin{abstract}
We describe a case of a 35-year-old woman who developed renal vasculitis in the setting of exposure to silicone after breast implantation.
\end{abstract}

\section{Introduction}

Silicone implantation is associated with scleroderma, systemic lupus erythematosus, and rheumatoid arthritis. In 1964, Miyoshi first coined the term human adjuvant disease in their report of two patients who developed connective tissue-like disease after exposure to silicone-related substances during augmentation mammoplasty. Since this first observation, there have been numerouspublished cases relating silicone exposure to autoimmunerheumatic diseases. We, herein, report a patient who developed vasculitis and chronic renal failure after implantation of silicone mammoplasty.

\section{Case Report}

A 35 years old woman with a history of a right mastectomy in December 2014, for ductal carcinoma in situ with immediate breast reconstruction with silicone prosthesis. The assessment of extension is normal. She was hospitalized fifteen days after surgery for urinary infection with discovery of renal failure with a creatinine level of $500 \mu \mathrm{mol} / \mathrm{L}$; she was put under antibiotic treatment and is then output with a creatinine of $200 \mu \mathrm{mol} / \mathrm{L}$. The patient was transferred to our department in late January 2015 for investigation of the renal failure (creatinine level $717 \mu \mathrm{mol} / \mathrm{l}$ ). Her physical examination was normal excluding significant edema of the lower members and normalarterial high blood pressure. Additional testing revealed microscopic hematuria, renal failure, elevated CRP and anaemia (Table 1).

Based on acute progressive renal failure with proteinuria and hematuria, kidney biopsy done revealed focal segmental necrotizing and pauci-immune crescentic glomerulonephritis, with moderate activity and minimal chronicity, minimal tubular atrophy, and interstitial fibrosis (Figure 1). Immunofluorescence microscopy was negative for any significant immunoglobulins and complement deposition. All immunological tests were negative: Anti Nuclear Antibody (ANA), cryoglobulins,cytoplasmic antibodies neutophilespolynuclear (ANCA), Anti Membranes Basal Glomerular (anti MBG). The thoracic scanner did not show a diffuse alveolar hemorrhage. Anechocardiogram showed severe left ventricular dysfunction with ejection fraction of $32 \%$. The silicone prosthesis was criminalized. Breast implant was removed. The patient was treated with a combination of pulses of methylprednisolone and cyclophosphamide. The evolution was unfavourable without improved renal function. The patient chose hemodialysis; immunosuppression was decreased and stopped.

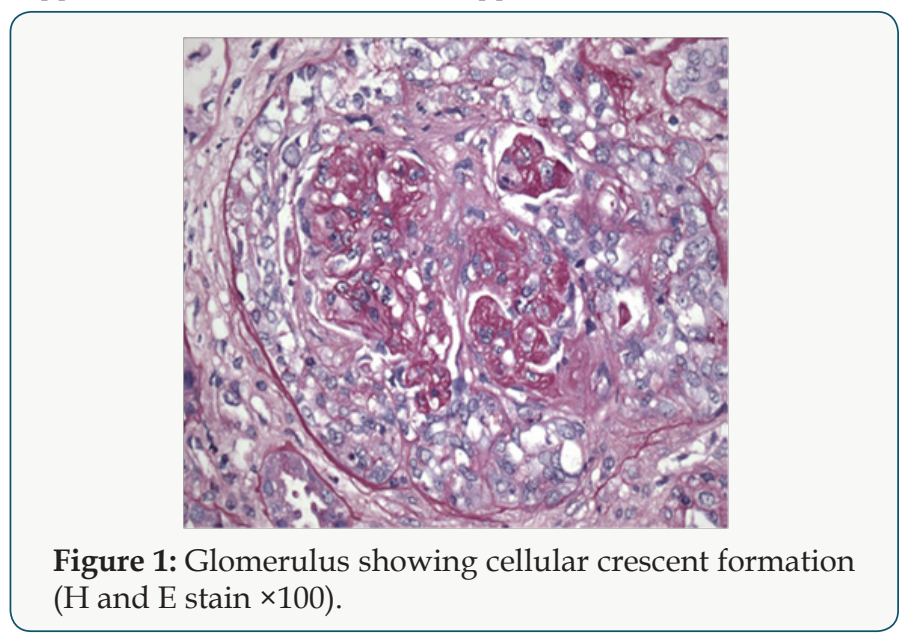


Table 1: Laboratory tests during hospital admission.

\begin{tabular}{|c|c|}
\hline Laboratory test & Result \\
\hline Hemoglobin $(\mathrm{g} / \mathrm{dL})$ & 7,4 \\
\hline White blood cell count $\left(\times 103 / \mathrm{mm}^{3}\right)$ & 4,5 \\
\hline Serum creatinine $(\mu \mathrm{moml} / \mathrm{L})$ & 1033 \\
\hline CRP $(\mathrm{mg} / \mathrm{dl})$ & 23 \\
\hline
\end{tabular}

\section{Discussion}

The relationship between silicone breast implants and autoimmune or connective-tissue diseases has been the focus of considerable medical and legal discussion throughout the past decade. Although evidence of a causal relationship between the implantation and the development of connective tissue disease is circumstantial, removal of the silicone prosthesis has been reported to result in subsequent remission [1]. Silicones are not biologically inert. Injectable as well as implantable silicones have proven capable of eliciting inflammatory and fibro proliferative responses [2]. Silicone exposure is associated with different systemic autoimmune diseases such as systemic lupus erythematosus, rheumatoid arthritis, progressive systemic sclerosis, and vasculitis [3]. We postulate that silicone in silicone-filled breast implants may increase the risk of developing (auto) immune diseases and immune deficiencies [3].

Silicone implants may cause local complications (such as capsular contracture, rupture, closed capsulotomy, gel "bleed", nodular foreign body granulomas in the capsular tissue and lymph nodes) or general symptoms. An adverse immune reaction with signs and symptoms of rheumatoid disorders is also possible [4]. The development of disease related to silicone implants would depend on genetic factors, so that only a very few women are potentially at risk. HLA-DR53 may be a marker of predisposed subjects [4]. From a public health perspective, breast implants appear to have a minimal effect on the number of women in whom connective-tissue diseases develop, and the elimination of implants would not be likely to reduce the incidence of connectivetissue diseases [5]. The mechanism of silica exposure in the development of small vessel vasculitis is not well understood but several potential mechanisms have been proposed [6]. One theory suggests that silica particles stimulate production of lymphocytes, including $\mathrm{T}$ cells and B cells, and that in certain clinical and genetic settings causes autoimmune disease as well as the production of auto antibodies, including ANCA [6]. A second theory suggests that silica particles activate monocytes and macrophages, resulting in the release of IL- 1 or tumour necrosis factor- $\alpha$, oxygen-derived free radicals, and lysosomal enzymes such as PR3 and MPO [6].

\section{Conclusion}

Silicone implantation is associated with scleroderma, systemic lupus erythematosus, and rheumatoid arthritis. This case report indicates the possibility of the development of small-vessel vasculitis after silicone breast implantation.

\section{References}

1. Haga HJ, Haaland P, Haga T (1992) Silicone breast implants and collagen diseases. Tidsskr Nor Laegeforen 112(6): 763-764.

2. Spiera RF, Gibofsky A, Spiera H (1994) Silicone gel filled breast implants and connective tissue disease: an overview. SJ Rheumatol 21(2): 239245.

3. Cohen Tervaert JW, Kappel RM (2013) Silicone implant incompatibility syndrome (SIIS): a frequent cause of ASIA (Shoenfeld's syndrome). Immunol Res 56(2-3): 293-298.

4. Iannello S, Belfiore F (1998) Silicone breast prosthesis and rheumatoid arthritis: a new systemic disease: siliconosis A case report and a critical review of the literature. Minerva Med 89(4): 117-130.

5. Esther C Janowsky, Lawrence L Kupper, Barbara S Hulka (2000) Metaanalyses of the relation between silicone breast implant and the risk of connective-tissue diseases. N Engl J Med 342(11): 781-790.

6. Judy Tan, FuadSpath, Rakesh Malhotra, Hamadeh Z, Acharya A (2014) Microscopic Polyangiitis following Silicone Exposure from Breast Implantation. Case Reports in Nephrology 2014: 1-3.
This work is licensed under Creative Commons Attribution 4.0 License

To Submit Your Article Click Here: Submit Articl

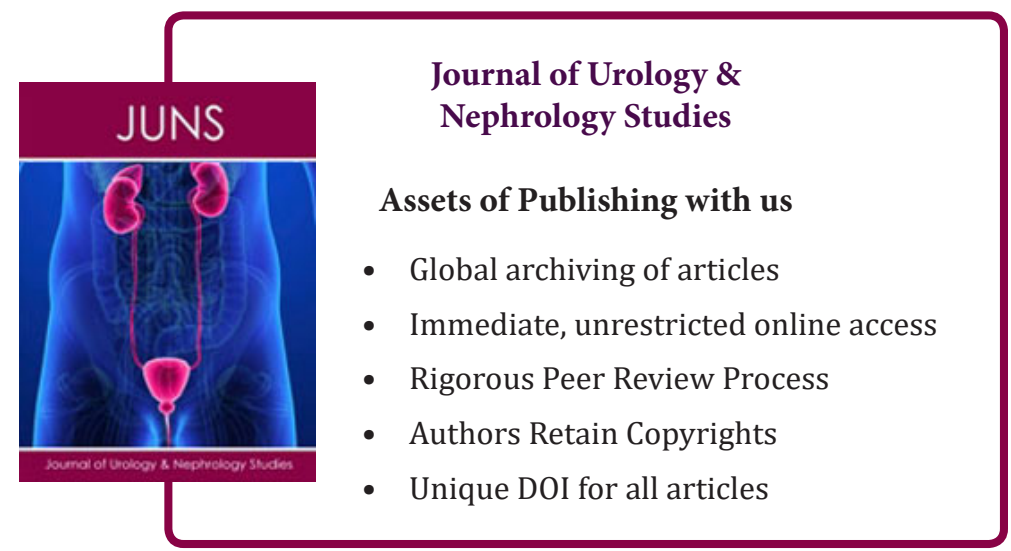

